\title{
Does community-based point of care HIV testing reduce late HIV diagnosis? A retrospective study in England and Wales
}

\author{
Nell Freeman-Romilly \\ Paula Sheppard \\ Sarika Desai \\ Nick Cooper \\ Michael Brady
}

\begin{abstract}
The objective of this study was to investigate if patients diagnosed in community clinics have higher baseline CD4 cell count than those diagnosed in GUM/HIV clinics. We undertook a retrospective review of baseline CD4 cell count for patients receiving a reactive HIV test in community-testing clinics. Eleven local HIV clinics were contacted to determine the baseline CD4 cell count of these patients. Baseline CD4 cell count of those diagnosed in the community was compared with mean local GUM/HIV clinic and median national baseline CD4 cell count for their year of diagnosis. Clients diagnosed in community settings had a mean baseline CD4 cell count of 481 cells/mm3 (SD 236 cells/mm3) and median baseline of 483 cells $/ \mathrm{m} 3$ (interquartile range $311-657$ ells $/ \mathrm{mm} 3$ ). This was significantly higher than those diagnosed in the GUM/HIV clinic local to the community-testing site (mean baseline CD4 397 cells/mm3, p⿳1/40.014) and the national median for that year $(336$ cells/mm3, p<0.001). HIV testing in community settings identifies patients at an earlier stage of infection than testing in clinical settings.
\end{abstract}

Keywords: Screening, AIDS, HIV, Diagnosis, Europe 


\section{INTRODUCTION}

Late diagnosis of HIV is an important predictor of morbidity, short-term mortality and HIV transmission ${ }^{1}$. In 2014, two fifths of HIV diagnoses in the UK were made 'late' (with a CD4 cell count $<350$ cells $/ \mathrm{mm}^{3}$ within three months of diagnosis) ${ }^{2}$.

Most HIV tests in the UK are undertaken in clinical settings. These include primary care, and antenatal clinics. Community HIV testing facilities are defined 'as those that are based outside pre-existing traditional healthcare settings. These include both stand-alone HIV testing services, provided separately from other clinical services, and venues primarily used for other purposes (such as social venues or community centres) where HIV testing is available as an additional service' ${ }^{3}$. Between 2006-2009 in the UK, by far the majority, 73\%, of all HIV diagnoses were made in sexual health clinics and less than $1 \%$ in non-clinical settings including prisons and the Blood Transfusion Service ${ }^{4}$

However, people cite overcrowding, waiting times, and the lack of anonymity as disincentives for attending sexual health clinics for HIV testing ${ }^{5}$. Black Africans in London cited further uncertainty about where to test and lack of time as barriers to testing $^{6}$. While, one UK study looking at the views of men who have sex with men (MSM), on STI testing in community settings, found that the majority would prefer to test for HIV in 'National Health Service (NHS) specialist service' settings ${ }^{7}$, studies around the world show that HIV testing outside of clinical facilities is acceptable and that mobile community HIV testing can be cost-effective in diagnosing new cases and 
effective at enrolling HIV positive patients in care before they become ill $^{8-13}$. A systematic review of forty-four community HIV testing programmes in resource-rich countries found that, 'community HIV testing strategies provide an acceptable alternative to HIV testing in healthcare settings and are feasible to implement' ${ }^{3}$.

Many of these studies were, however, undertaken in either developing world settings or the United States. Neither of these environments accurately reflects the state funded and free at the point of use healthcare system found in the UK. A US study found that clients testing in the community were older and more likely to be HIV-positive than those testing in clinical settings ${ }^{14}$, while a small UK study ${ }^{15}$ and most developing world studies found the reverse.

Terrence Higgins Trust (THT) is the largest HIV and Sexual Health charity in the UK and has run community HIV testing programs since 2004. THT community HIV testing clinics have performed more than 14,000 HIV tests since 2008 in England and Wales. These nurse-led or Healthcare Assistant delivered clinics are located in easily accessible sites including high street offices, saunas, libraries, and churches and often operate on evenings and weekends. A small study evaluating one such service found that the average baseline CD4 count was higher, although not significantly so, when compared to clients of the local GUM/HIV clinic $^{15}$. For the period 2008 to 2012 we used data from THT's community HIV testing clinics to determine whether people receiving a reactive HIV test at a community-testing site were identified at a different stage of HIV infection 
compared with those diagnosed in clinical settings and national averages. We refer to these clinical settings as GUM/HIV clinics due to the integrated nature of many UK sexual health services. Patients may present for HIV testing at a primary healthcare or GUM clinic but baseline CD4 data is recorded and provided by a hospital HIV clinic. We defined a patient's first recorded CD4 count as their baseline CD4 count.

\section{Objectives}

1) To determine if newly diagnosed patients receiving a reactive point-of-care HIV test in a community setting have different baseline CD4 counts when compared with patients diagnosed in clinical settings.

2) To explore the care pathway of people testing for HIV in community-clinics, including what proportion of patients engages with subsequent clinical care.

\section{METHODS:}

We received ethical approval from South East Coast - Kent NRES Committee as project 12/LO/1778. Following this, we searched THT's database and paper records to identify people who had received a reactive HIV test in a community clinic between 2008 and 2012. We contacted the hospital specified on each patient's care pathway to determine if they had accessed subsequent HIV care and, if so, to establish their baseline CD4 count. We provided an encrypted list of the patients' Soundex (scrambled surname and date of 
birth code) to Public Health England (PHE) to ascertain whether patients had sought HIV care elsewhere in England or Wales and, if so, what had been their baseline CD4 count. PHE were also able to identify whether patients had received a CD4 count pre-dating their visit to THT's Community Clinic.

\section{Study Population}

Figure 1 shows the flow chart of how we arrived at our final sample of 74 patients who were newly diagnosed with HIV in a community clinic and had a known baseline CD4 count.

Figure 1 here

A demographic breakdown of the sample is given in table 1.

\begin{tabular}{lllll}
\hline Total patients & Total & Patients with & Newly \\
tested in & reactive & new HIV & diagnosed \\
community & population in & diagnoses in & patients with \\
clinics & community & community & CD4 count \\
& $(\mathrm{n}=10560)(\%)$ & clinics & clinics & originally \\
& & $(\mathrm{n}=214)(\%)$ & $(\mathrm{n}=36)(\%)$ & tested in \\
& & & & community \\
& & & & clinics $(\mathrm{n}=74)$ \\
\hline Ethnicity & & & & $(\%)$ \\
\hline White UK & & & & \\
\hline
\end{tabular}




\begin{tabular}{|c|c|c|c|c|}
\hline White non-UK & $562(5)$ & $16(7)$ & $2(6)$ & $9(12)$ \\
\hline Black African & $2721(26)$ & $80(37)$ & $23(64)$ & $12(16)$ \\
\hline Other Black including & $957(9)$ & $11(5)$ & $2(6)$ & $3(4)$ \\
\hline \multicolumn{5}{|l|}{ mixed } \\
\hline Asian and mixed & $795(8)$ & $7(3)$ & $1(3)$ & $1(4)$ \\
\hline Ethnicity Unknown & $426(4)$ & $11(5)$ & $2(6)$ & $0(0)$ \\
\hline \multicolumn{5}{|l|}{ Sexuality } \\
\hline Heterosexual & $6840(65)$ & $96(45)$ & $26(72)$ & $16(22)$ \\
\hline Homosexual & 2039 (19) & $82(38)$ & $4(11)$ & $51(69)$ \\
\hline Bisexual & $477(5)$ & $13(13)$ & $3(8)$ & $5(7)$ \\
\hline Sexuality Unknown & $1207(11)$ & $23(23)$ & $3(8)$ & $2(3)$ \\
\hline \multicolumn{5}{|l|}{ Gender } \\
\hline Female & $3059(29)$ & $66(31)$ & $19(53)$ & $10(14)$ \\
\hline Male & $7504(71)$ & $148(69)$ & $17(47)$ & $64(86)$ \\
\hline Median age & 29 & 32 & 36 & 35 \\
\hline
\end{tabular}

\footnotetext{
Mean Baseline CD4 count for patients diagnosed 481 (SD 236) (vs Mean Baseline CD4 count for in community setting patients diagnosed in referral hospital $p=0.014)$

Mean Baseline CD4 count for patients diagnosed 397 (SD 34)

in referral hospital

Median Baseline CD4 count for patients $\quad 483$ (311-657)

diagnosed in a community setting (vs Public Health England's national Median

(IQR) $\quad$ Baseline CD4 count $\mathrm{p}<0.001)$

Public Health England's national Median 336

Baseline CD4 count (IQR not available)

Median number of days between reactive $3(1-9)$

Community Test and presenting to a hospital
} 
HIV clinic for a CD4 Test in newly diagnosed

patients (IQR)

Median number of days between reactive

$2(1.5-3)$

Community Test and presenting to a hospital

HIV clinic for a CD4 Test in patients with

identified pre-existing HIV diagnosis (IQR)

Table 1. Demographics of patients testing in HIV community clinics, those who received a reactive test, those who had received an HIV diagnosis prior to their community clinic test and those who were newly diagnosed in the community clinic and could be traced to a baseline CD4 count

\section{Statistical methods}

Comparator CD4 counts were obtained from the hospital HIV clinics where THT community-testing patients went for confirmatory tests and care. These clinics provided annual mean averages of their patients' baseline CD4 counts from 2008 to 2012.

Public Health England (PHE) provided national median CD4 counts from 2008 to 2011. National median CD4 counts for 2012 were not available at the time of data analysis. These, however, showed consistency between years, and clients who were tested in 2012 were assigned the PHE value from 2011. Medians are used instead of means here because extreme outliers are found at the national level and these skew the mean values. There were no significant outliers in the THT data we collected and so we compared means for this part of the study. 
T-tests were performed to compare the mean baseline CD4 count of the communitytesting clients who presented for hospital HIV care to the mean baseline CD4 count for the HIV service to which they were referred, in the year they presented. PHE provided national median baseline CD4 counts. We performed Wilcoxon signed rank tests to compare community tested clients to the national median from PHE for the year they were tested.

To control for confounders, we performed further tests where individuals were categorised by gender, sexuality and ethnicity, and compared with clinic and national level medians for the same demographics. Due to very small sample sizes in the demographic-specific tests, we pooled these into a meta-analysis to estimate an overall effect.

We performed further t-tests to ascertain if community-testing clients were more likely to be diagnosed at a CD4 count $>350$ or $>500$ cells $/ \mathrm{mm}^{3}$ compared to those testing in clinical settings. These figures were chosen as they were the recommended CD4 counts at which patients were advised to start treatment in the UK and the US respectively at the time of testing. Finally, we performed a binary logistic analysis to examine which demographic factors predicted whether an individual presented for a follow-up appointment.

\section{RESULTS}


Two-hundred-and-fourteen people received a reactive HIV diagnosis in the study period (2.0\%). Seventy-four (36\%) fit the inclusion criteria of being newly diagnosed and subsequently confirmed as accessing HIV treatment in England or Wales. Thirty-six patients were already aware they were HIV positive at the time of their community-clinic test and 104 patients could not be linked to a subsequent baseline CD4 count. The mean baseline CD4 count of patients who tested positive in a THT community clinic was 84 cells $/ \mathrm{mm}^{3}$ higher than that of patients diagnosed at the referral hospitals' GUM/HIV clinics $(\mathrm{p}=0.014)($ see table 1$)$.

Wilcoxon signed rank tests showed that community-tested patients were significantly more likely to have higher CD4 counts than the national population. Fifty-nine community-tested patients had CD4 counts higher than the national median while twenty presented below. Clients diagnosed in community clinics were significantly more likely to be diagnosed at a baseline CD4 >350 cells $/ \mathrm{mm}^{3}$ than clients diagnosed in clinical settings. This effect was not replicated for baseline CD4 count $>500$ cells $/ \mathrm{mm}^{3}$. T-tests controlling for sexuality, ethnicity, and gender found that men who have sex with men (MSM), diagnosed in community settings, had significantly higher baseline CD4 counts than clients tested in clinical settings and were significantly more likely to be diagnosed at a baseline $\mathrm{CD} 4>350$ cells $/ \mathrm{mm}^{3}$.

Figure $2 \mathrm{~A}$ forest plot showing the difference in the mean baseline CD4 count between those tested in a THT community-clinic and the national median for each demographic group. Odds ratios are given on the $\mathrm{X}$-axis.

Figure 2 here 
Table 2 shows the results of a binary logistic regression analysis testing the demographic predictors of follow-up likelihood. The most important demographic predictor is being homosexual or bisexual (these categories were pooled in order to compare this group to heterosexual participants). MSM are almost four times more likely to present for followup after a reactive test in a community-clinic. Being male and white were also associated with higher odds of presenting for follow-up although these results are not statistically significant. Age was not a predictor of likely follow-up.

\begin{tabular}{lllll}
\hline Follow-up & Odds ratio & P-value & \multicolumn{2}{c}{ 95\% CI } \\
\hline Male (ref female) & 1.73 & 0.285 & 0.63 & 4.73 \\
MSM (ref & 3.84 & 0.015 & 1.29 & 11.40 \\
heterosexual) & & & & \\
White (ref. non- & 1.97 & 0.208 & 0.69 & 5.65 \\
white) & & & & \\
Age at test & 1.00 & 0.823 & 0.97 & 1.04 \\
\hline
\end{tabular}

Table 2 Results from binary logistic regression analysis of follow-up. Odds ratios, significance tests and $95 \%$ confidence intervals are presented for demographic indicators of the odds of presenting at follow-up after THT community-testing. An odds ratio of higher than one denotes higher odds of follow-up.

\section{Previously diagnosed patients and re-engagement}


Thirty-six people were already aware that they were HIV positive when they took an HIV test at a community-clinic. Of these, six patients were confirmed as subsequently accessing specialist HIV services having never, or not within the last 2 years, received HIV care in the UK. They sought care a median of 2 days (IQR 1.5 to 3 ) following their community HIV test. Sixteen patients informed the nurse at the community clinic that they were aware of their positive HIV status. Notes in the patients' records showed that nurses counselled previously diagnosed patients about the importance of accessing HIV treatment and gave advice on how to access care. Twenty patients had a pre-existing baseline CD4 count, which was taken at a median of 1010 days (IQR 431-1859) before their community clinic HIV test.

\section{DISCUSSION}

Our results show that patients receiving an HIV diagnosis through community-testing are, on average, diagnosed at an earlier stage of infection when compared with patients testing in clinical settings. They are also significantly more likely to be diagnosed at a baseline CD4 above 350 cells $/ \mathrm{mm}^{3}$. These associations are significant for the aggregated patient group and for MSM patients. It is not significant for Black African or heterosexual clients, but the average CD4 count at baseline was still higher when tested in community settings, compared with clinical settings. In our study, only a small number of Black African and heterosexual clients could be linked to a baseline CD4 count, and the small sample size is likely to contribute to this lack of statistical significance. Those that presented to a hospital for confirmatory testing and care presented a median of 3 days 
after their community HIV test. This falls within BHIVA's 2013 Standard of Care guidelines that indicate that all patients should be offered a full baseline assessment, including a CD4 count test, within two weeks of diagnosis with $\mathrm{HIV}^{16}$. It is possible that people diagnosed in community settings are more likely to access care sooner after diagnosis than those in clinical settings and will therefore appear have a higher baseline CD4 count. A 2014 study found that adults who received their HIV diagnosis in primary care or a community setting were significantly less likely to have presented for HIV care within one month, compared to those who were diagnosed in an integrated GUM/HIV clinic $76 \%$ vs $91 \%$, p0.001) ${ }^{17}$.

\section{Access to Care}

One hundred and four (49\%) clients could not be confirmed as having accessed HIV care therefore we were unable to link them to a baseline CD4 count. This could reflect some patients using false names or dates of birth when testing in community clinics in addition to those patients who have yet to seek HIV care. It may also represent some clients with false reactive HIV point-of-care-tests that were not subsequently confirmed as HIV positive and therefore did not have a baseline CD4 count. Binary logistic regression analysis found that being male and white increased the likelihood of being linked to follow-up however these results are not statistically significant, and there was no effect of age. MSM clients were almost four times more likely to be linked to follow-up care compared with heterosexual clients. Most of the heterosexual men who had reactive tests identified themselves as of African descent although we are unable to ascertain when they had migrated to the UK. 
Difficulty in maintaining referral pathways is an established challenge in communitytesting ${ }^{18}$. This is possibly because while community-testing removes barriers to HIV testing, these barriers remain in place when it is necessary for the patient to access hospital based HIV care. Community-testing may allow clients to test earlier than they otherwise would have done, but this does not mean they will be ready to accept their HIV diagnosis any sooner. The barriers a person needs to overcome to access clinic-based testing may mean they are more motivated to test and will be more prepared to proceed to seeking care.

In previous studies on community HIV testing, a similar proportion of reactive patients were confirmed to have accessed HIV care ${ }^{19}$. Studies in the United States, found that $48 \%$ of the patients who received a reactive HIV test result in South Carolina and $77 \%$ in New York City linked to care within three months of their test ${ }^{20-21}$. A systematic review on HIV testing in community setting found only three studies collected data on the proportion of HIV diagnosed patients who transferred to care, and that these rates were $75-100 \%$ of all diagnosed patients ${ }^{3}$. All three studies were based in saunas and provided community HIV testing to MSM only ${ }^{15,22-23}$.

Other studies have demonstrated that access to and retention in HIV care varies between demographic groups. A South London HIV clinic investigated the loss to follow up among its patients. It found that while more than a quarter of the heterosexual male black 
African (26.2\%) and Caribbean (29.3\%) patients were lost to all UK HIV care and follow up, this occurred with less than $18 \%$ of white and black MSM and white heterosexual male and female patients ${ }^{24}$.

A study of Black Africans in the UK found that "fear of stigmatisation, deportation and expectations of HIV as incurable or as a 'death sentence' continue to deter respondents from taking an HIV test" ${ }^{\text {"6 }}$. Black African workshop participants requested that community HIV testing staff should have training on, '1) dealing with questions about immigration status and entitlement to HIV treatment; [and] 2) discussing prevention of mother-to-child-transmission...'25. These examples suggest that HIV positive black African patients may have different needs and health seeking behaviours than MSM patients, despite often being combined into the same discussion on the utility and outcomes of of community HIV testing programmes.

Terrence Higgins Trust has a long history of working with gay men and is well accepted within the UK MSM community. It is possible that THT's community clinics are well designed for the MSM community but need to better understand the needs of the Black African community to increase progression from HIV testing into treatment.

\section{Pre-diagnosed patients}

Thirty-six patients were aware of their positive HIV status before their reactive community clinic test. Nearly $41 \%$ of these patients told the THT staff of their positive 
HIV status. Community-testing can be used to identify undiagnosed patients, and to provide a supportive opportunity to re-engage patients with specialist HIV care.

This study's findings were consistent across all five years, all demographic groups, and all referral clinics. Participants came from a range of urban and peri-urban settings in England and Wales. It is therefore likely that these findings are generalisable to other developed world settings with free-at-point-of-use health care systems. Further research is needed to understand how to improve the number of confirmed patients seeking care following a reactive HIV test in a community-clinic. This is particularly necessary for Black African patients. In recent years THT's community clinics have strengthened their referral pathways into local HIV services and now ensure that all patients with a reactive result are followed up at 2 weeks to ensure access-to-care. It would be useful to understand how community clinics are used to provide entry or re-engagement points into the medical system, as well as in counselling existing patients, normalising HIV testing, and providing health promotion opportunities for HIV-negative patients.

\section{STRENGTHS AND LIMITATIONS}

The number of patients who had a reactive HIV test in the community, had never previously been diagnosed with HIV, and for whom we were able to establish a baseline CD4 count is a small proportion of the total number of reactive community HIV tests. The impact of the missing data on the validity of the study findings makes it difficult to 
conclusively state that increasing the availability of community testing would increase the national average baseline CD4 count at diagnosis.

This study was dependent on patients providing the community-testing site with the same information they used if they sought HIV care. If they provided different information we were unable to link their baseline CD4 count. Patients who provided inconsistent information between HIV services may differ from those who provided consistent information. Hospitals listed on the local THT care pathway are geographically near to the community clinic. Patients more fearful of stigma may have been more likely to seek HIV care away from their local area and not attend the referral hospital. We ameliorated this limitation by collaborating with PHE to obtain baseline CD4 counts from HIV clinics across the UK. Due to incomplete and delayed reporting, PHE did not have all the clinic baseline CD4 counts that we had obtained. There may be other patients receiving HIV care but are missing from PHE's national baseline CD4 database. This would be a source of bias as it excludes some of the clients who sought HIV care away from their local area. We did not have national data for 2012 and used national data from 2011 as a proxy as we did not expect there to be much change in the median national baseline CD4 count between 2011 and 2012 .

This study does not investigate why patients appear to be testing at community clinics at an earlier stage of HIV infection than those testing in clinical settings. It may be that patients prefer the same day test results offered in community clinics, compared to having to wait for results when testing in a primary or hospital based setting. It may be that 
community clinics avoid having to provide the documentation that is required to register with a GP or that could be linked to other personal records. A minority of patients testing in clinical settings are being tested in A and $\mathrm{E}$ or as part of a medical admission. Their hospital attendance may be because they are symptomatic with an HIV related condition and these patients may never have tested for HIV until the point at which they became unwell. We suggest that all of these could be areas for further research.

Nevertheless, this study is the first to test whether HIV positive community-tested patients are diagnosed at a higher baseline CD4 count, than those who are tested in clinical settings. Although this study needs to be replicated with more data, our results suggest that this is indeed the case.

\section{CONCLUSION}

Our findings suggest that increasing the scale of community-based HIV testing would serve the varying health needs of certain populations at high risk of HIV and contribute to reducing the proportion of late HIV diagnoses in the UK. In every category, we found that community-tested patients had higher CD4 counts than those who first tested in a hospital. Early diagnosis is crucial for the greatest benefits of treatment to be achieved. It is futile, however, to give someone an HIV diagnosis without providing them with the support they need to access treatment and this should be central to all community-testing programs. 
Key Messages

- Community HIV testing identifies patients at a significantly higher CD4 count than testing in conventional settings.

- Community HIV testing provides useful services to those already diagnosed with HIV who may not be accessing care

- Clients diagnosed with HIV in a community setting need culturally appropriate support to ensure they access HIV care.

\section{ACKNOWLEDGMENTS}

We are grateful to Sarah Crawley, Jarrad Richards, Iain Reeves, Ashini Jayasuriya, Humphrey Birley, Stewart Attridge, Mark Gompels, Louise Jennings, Jonathan Roberts, Julie Field, Amy Evans, Lisa Murthen, Cheryl Tawana for supplying us with data. We thank Rachel Baggaley and Bernadette Hensen for assisting us with the literature review. We also thank all the participants without whom this study would not have been possible. This study was funded by The Society for General Microbiology, SGM Elective Grants $2012-\mathrm{E} 12 / 7$.

The authors declare no competing interests. Nell Freeman-Romilly previously worked with Terrence Higgins Trust (2008-2011). Michael Brady is Medical Director of Terrence Higgins Trust. 


\section{Bibliography}

1. Farnham P, Gopalappa C, Sansom S, et al. Updates of Lifetime Costs of Care and Quality-of-Life Estimates for HIV-Infected Persons in the United States. JAIDS Journal of Acquired Immune Deficiency Syndromes. 2013;64(2):183-189.

2. The HIV and AIDS Reporting Team, HIV and STI Department, (2015). HIV new diagnoses, treatment and care in the UK 2015. [online] Public Health England, p.4. Available at:

https://www.gov.uk/government/uploads/system/uploads/attachment_data/file/469405/HI V_new_diagnoses_treatment_and_care_2015_report20102015.pdf [Accessed 18 Oct. 2016].

3. Thornton, A., Delpech, V., Kall, M. and Nardone, A. (2012). HIV testing in community settings in resource-rich countries: a systematic review of the evidence. $H I V$ Medicine, 13(7), pp.416-426.

4. Kall, M., Thornton, A., Nardone, A. and Delpech, V. (2011) Where do we diagnose HIV? Monitoring new diagnoses made in non-traditional settings. 17th annual British HIV Association conference, Bournemouth, abstract P161, 2011.

5. Llewellyn C, Pollard A, Miners A, et al. Understanding patient choices for attending sexually transmitted infection testing services: a qualitative study. Sexually Transmitted Infections. 2012;88(7):504-509. 
6. Health Protection Agency. Mayisha II main study report Assessing the feasibility and acceptability of community based prevalence surveys of HIV among black Africans in England. 2005. London: Health Protection Agency; 2005 p. 4, 6, 8.

7. Horwood, J., Ingle, S., Burton, D., Woodman-Bailey, A., Horner, P. and Jeal, N. (2015). Sexual health risks, service use, and views of rapid point-of-care testing among men who have sex with men attending saunas: a cross-sectional survey. International Journal of STD \& AIDS, 27(4), pp.273-280.

8. Sweat M, Morin S, Celentano D, et al. Community-based intervention to increase HIV testing and case detection in people aged 16-32 years in Tanzania, Zimbabwe, and Thailand (NIMH Project Accept, HPTN 043): a randomised study. The Lancet Infectious Diseases. 2011;11(7):525-532.

9. Grabbe K, Menzies N, Taegtmeyer M, et al. Increasing Access to HIV Counseling and Testing Through Mobile Services in Kenya: Strategies, Utilization, and CostEffectiveness. JAIDS Journal of Acquired Immune Deficiency Syndromes. 2010;54(3):317-323. 
10. Wachira J, Kimaiyo S, Ndege S, et al. What Is the Impact of Home-Based HIV Counseling and Testing on the Clinical Status of Newly Enrolled Adults in a Large HIV Care Program in Western Kenya?. Clinical Infectious Diseases. 2011;54(2):275-281.

11. Lahuerta M, Sabido M, Giardina F, et al. Comparison of users of an HIV/syphilis screening community-based mobile van and traditional voluntary counselling and testing sites in Guatemala. Sexually Transmitted Infections. 2010;87(2):136-140.

12. MacPherson, P., Chawla, A., Jones, K., Coffey, E., Spaine, V., Harrison, I., Jelliman, P., Phillips-Howard, P., Beynon, C. and Taegtmeyer, M. (2011). Feasibility and acceptability of point of care HIV testing in community outreach and GUM drop-in services in the North West of England: A programmatic evaluation. BMC Public Health, 11(1).

13. Manavi, K., Williams, G. and Newton, R. (2012). The uptake of HIV and syphilis testing in a nurse-delivered service during Gay Pride events. International Journal of STD \& AIDS, 23(12), pp.887-889.

14. Smith L, Rudy E, Javanbakht M, et al. Client Satisfaction with Rapid HIV Testing: Comparison Between an Urban Sexually Transmitted Disease Clinic and a CommunityBased Testing Center. AIDS Patient Care and STDs. 2006;20(10):693-700. 
15. Bailey A, Roberts J, Weatherburn P, et al. Community HIV testing for men who have sex with men: results of a pilot project and comparison of service users with those testing in genitourinary medicine clinics. Sexually Transmitted Infections. 2008;85(2):145-147.

16. British HIV Association, (2012). Standards of care for people living with HIV in 2013. [online] London: British HIV Association. Available at: http://www.bhiva.org/standards-of-care-2013.aspx/ [Accessed 30 Sep. 2016].

17. Yin, Z., Brown, A., Hughes, G., Gill, O. and Delpech, V. (2014). HIV in the United Kingdom 2014 Report: data to end 2013. London: Public Health England, p.14.

18. Pedrana A, Stoove M, Bowring A, et al. P5-S7.17 Accepting the good with the bad: "barriers and facilitators of community-based HIV testing services for gay men: a systematic review". Sexually Transmitted Infections. 2011;87(Suppl 1):A349-A349.

19. Govindasamy D, van Schaik N, Kranzer K, et al. Linkage to HIV Care From a Mobile Testing Unit in South Africa by Different CD4 Count Strata. JAIDS Journal of Acquired Immune Deficiency Syndromes. 2011;58(3):344-352.

20. Tripathi A, Gardner L, Ogbuanu I, et al. Predictors of time to enter medical care after a new HIV diagnosis: a statewide population-based study. AIDS Care. 2011;23(11):13661373. 
21. Jenness S, Myers J, Neaigus A, et al. Delayed entry into HIV medical care after HIV diagnosis: Risk factors and research methods. AIDS Care. 2012;24(10):1240-1248.

22. Lister, N., Smith, A., Tabrizi, S., Garland, S., Hayes, P. and Fairley, C. (2005). Comprehensive clinical care on-site in men-only saunas: confidential STI/HIV screening outreach clinic. International Journal of STD \& AIDS, 16(12), pp.794-798.

23. Daskalakis, D., Silvera, R., Bernstein, K., Stein, D., Hagerty, R., Hutt, R., Maillard, A., Borkowsky, W., Aberg, J., Valentine, F. and Marmor, M. (2009). Implementation of HIV Testing at 2 New York City Bathhouses: From Pilot to Clinical Service. Clinical Infectious Diseases, 48(11), pp.1609-1616.

24. Gerver, S., Chadborn, T., Ibrahim, F., Vatsa, B., Delpech, V. and Easterbrook, P. (2010). High rate of loss to clinical follow up among African HIV-infected patients attending a London clinic: a retrospective analysis of a clinical cohort. Journal of the International AIDS Society, 13(29).

25. Prost, A., Sseruma, W., Fakoya, I., Arthur, G., Taegtmeyer, M., Njeri, A., Fakoya, A. and Imrie, J. (2007). HIV voluntary counselling and testing for African communities in London: learning from experiences in Kenya. Sexually Transmitted Infections, 83(7), pp.547-551. 